\title{
FRAMES-2.0 Software System: Frames 2.0 Pest Integration (F2PEST)
}

\section{KJ Castleton}

PD Meyer

May 2009

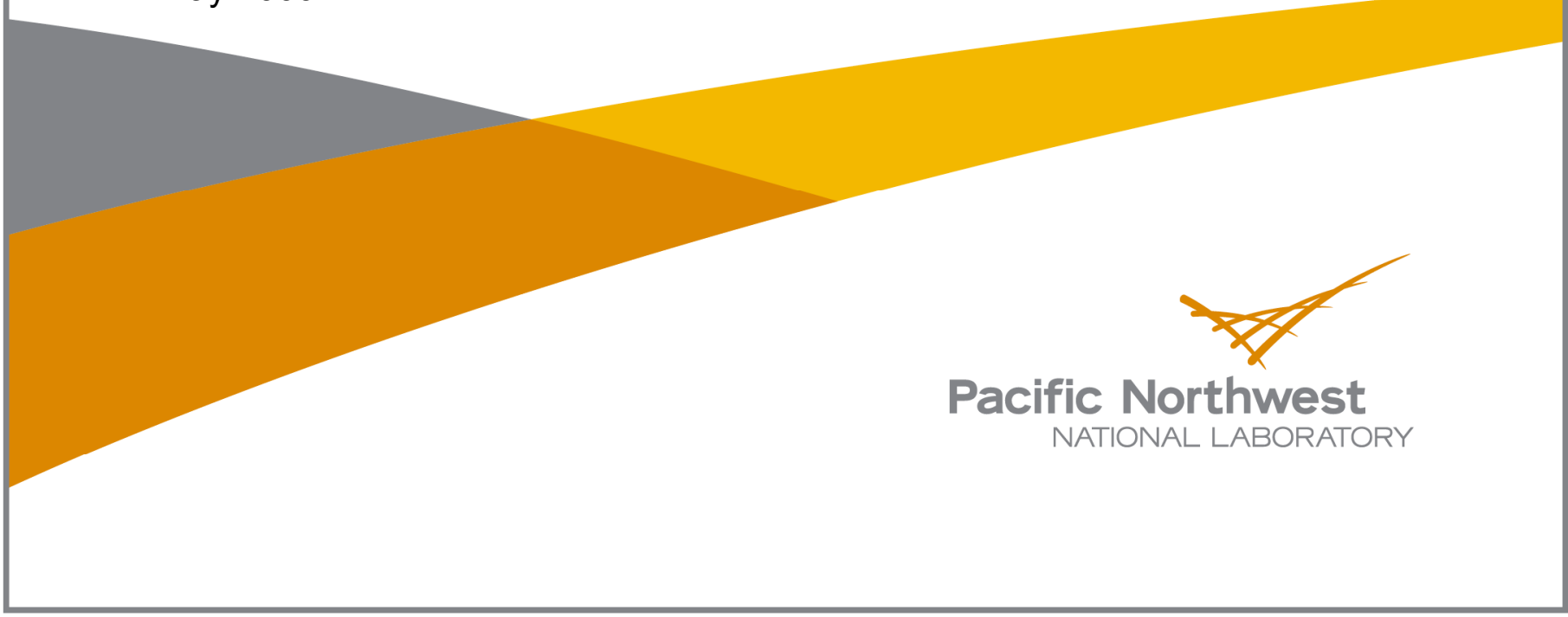




\title{
DISCLAIMER
}

This report was prepared as an account of work sponsored by an agency of the United States Government. Neither the United States Government nor any agency thereof, nor Battelle Memorial Institute, nor any of their employees, makes any warranty, express or implied, or assumes any legal liability or responsibility for the accuracy, completeness, or usefulness of any information, apparatus, product, or process disclosed, or represents that its use would not infringe privately owned rights. Reference herein to any specific commercial product, process, or service by trade name, trademark, manufacturer, or otherwise does not necessarily constitute or imply its endorsement, recommendation, or favoring by the United States Government or any agency thereof, or Battelle Memorial Institute. The views and opinions of authors expressed herein do not necessarily state or reflect those of the United States Government or any agency thereof.

\author{
PACIFIC NORTHWEST NATIONAL LABORATORY \\ operated by \\ BATTELLE \\ for the \\ UNITED STATES DEPARTMENT OF ENERGY \\ under Contract DE-AC05-76RL01830
}

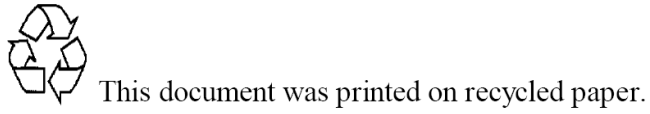

$(9 / 2003)$ 


\section{FRAMES-2.0 Software System: Frames 2.0 Pest Integration (F2PEST)}

Karl J. Castleton

Philip D. Meyer

May 2009

Prepared for The U.S. Nuclear Regulatory Commission

Office of Nuclear Regulatory Research

Division of Systems Analysis \& Regulatory Effectiveness

Washington, DC 20555-0001

under a Related Services Agreement with

the U.S. Department of Energy

Contract DE-AC05-76RL01830

Pacific Northwest National Laboratory

Richland, Washington 99352 



\section{Summary}

The implementation of the FRAMES 2.0 F2PEST module is described, including requirements, design, and specifications of the software. This module integrates the PEST parameter estimation software within the FRAMES 2.0 environmental modeling framework. A test case is presented. 



\section{Contents}

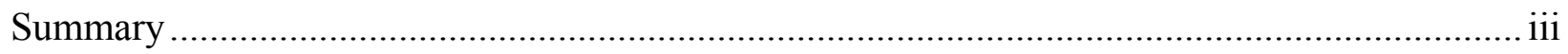

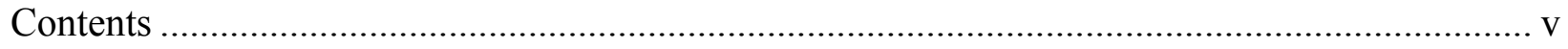

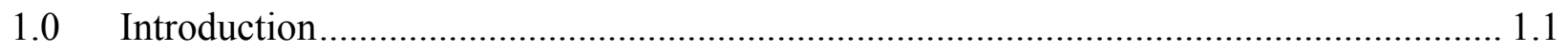

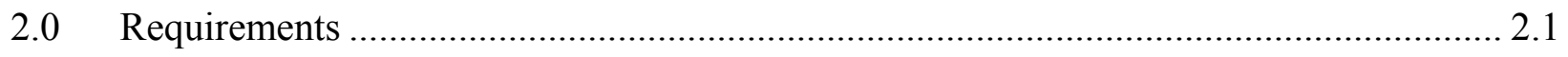

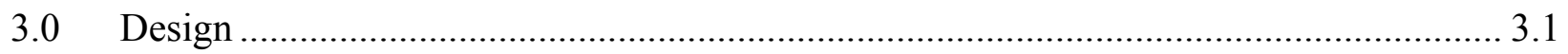

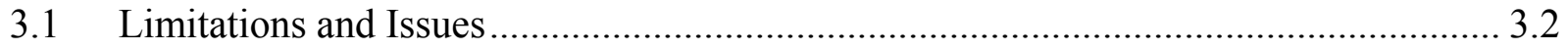

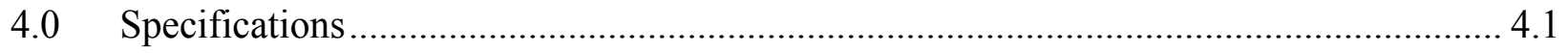

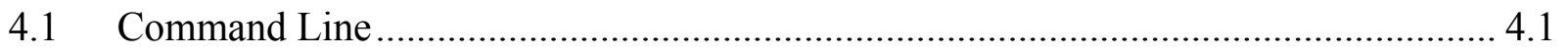

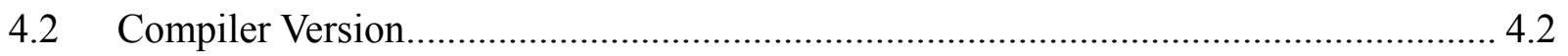

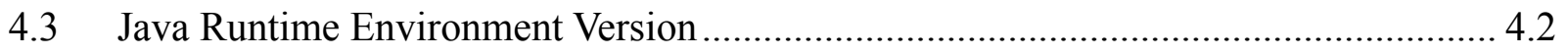

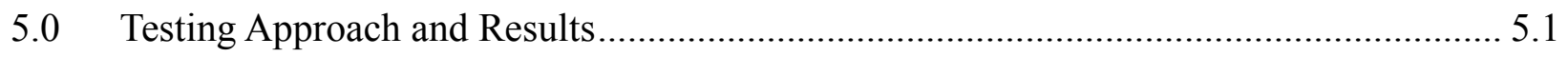

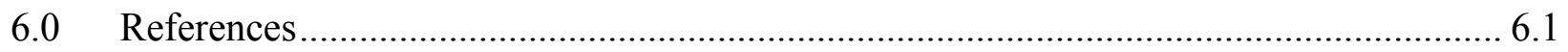

\section{Tables}

Table 1-1. Example model input file and corresponding PEST template file.............................. 1.2

Table 1-2. Example model output file and corresponding PEST instruction file ........................ 1.3

Table 4-1. Dictionaries produced by F2PEST: ParameterEvaluation containing parameter estimation results and MultiModelComparison containing model comparison statistics .... 4.3

Table 5-1. Solution concentrations from Eq. 2 and randomly perturbed observations for use in

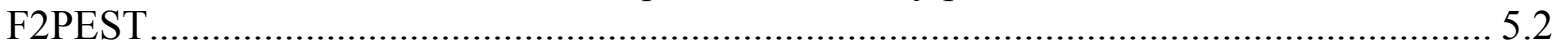

Table 5-2. PEST files generated by F2PEST (final state) ......................................................... 5.5 


\section{Figures}

Figure 2-1. An example FRAMES 2.0 diagram that includes F2PEST modules ..................... 2.2

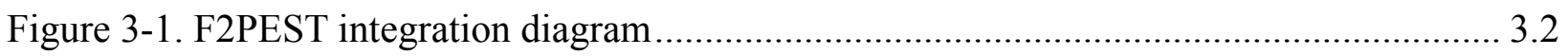

Figure 5-1. Calculated solution to Eq. 2 and randomly perturbed observation values used in

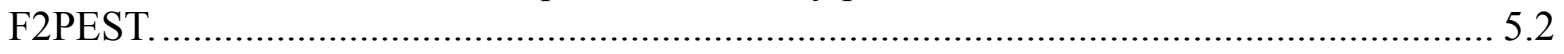

Figure 5-2. Simulation editor in FRAMES illustrating the F2PEST test case......................... 5.3

Figure 5-3. Parameter selection tab from the F2PEST UI ................................................ 5.4

Figure 5-4. Observation selection tab from the F2PEST UI............................................... 5.4

Figure 5-5. PEST control file tab from the F2PEST UI..................................................... 5.5

Figure 5-6. ParameterEvaluation dictionary produced by F2PEST including parameter estimates

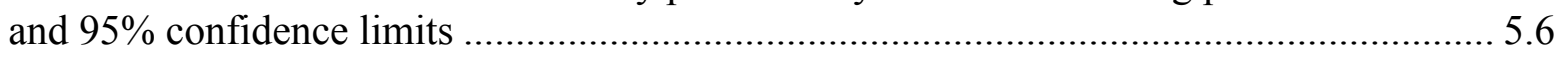

Figure 5-7. ParameterEvaluation dictionary produced by F2PEST including parameter covariance and correlation matrices

Figure 5-8. MultiModelComparison dictionary produced by F2PEST 5.8 


\subsection{Introduction}

The U.S. Nuclear Regulatory Commission (NRC) staff members are responsible for reviews of Early Site Permit (ESP) and Combined License (COL) applications for new nuclear power plants. Using site-specific information from the ESP and COL applications, NRC staff draft environmental impact statements (EIS) and perform confirmatory safety-related analyses for these proposed new plants. Many important technical-review issues surround the assessment of site-specific ground-water and surface-water conditions and behaviors that influence site hydrology and radionuclide transport. Under a variety of reasonable release scenarios, radionuclide transport through the ground-water pathway is a key component in assessing potential exposures to the public. Technical review issues related to groundwater transport of radionuclides include the formulation of hydrogeologic site conceptual models, the identification of plausible radionuclide transport pathways, the appropriate degree of complexity and conservatism in modeling transport, and the adequacy of site characterization data and monitoring plans. Resolution of these issues can be facilitated by a systematic effort to acknowledge and assess the important uncertainties in the characterization and representation of site-specific groundwater transport.

A systematic and quantitative approach for combining estimates of uncertainties in the data, conceptual models, parameters, and scenarios related to hydrogeology was presented by Meyer et al. (2007). An application to uranium transport at the U.S. Department of Energy Hanford Site 300 Area illustrated the practical benefits of the approach to provide better estimates of predictive uncertainty, quantitative results for use in assessing risk, and an improved understanding of the system behavior and limitations of the models. Although the approach and its applications were specifically designed for reviews of radionuclide transport at complex decommissioning sites, the approach is applicable to any analysis involving hydrologic modeling, including assessing nuclear facility siting, designing ground-water monitoring programs, remediating ground-water, and identifying and selecting strategies to preclude offsite migration of abnormal radionuclide releases.

To facilitate application of a systematic approach to uncertainty assessment in ESP and COL reviews, some of the methods described in Meyer et al. (2007) have been integrated with the Framework for Risk Analysis in Multimedia Environmental Systems (FRAMES) version 2 software. FRAMES ${ }^{1}$ is a software platform that allows users the ability to select and implement environmental software models for risk assessment and management problems. This program is a flexible and holistic approach to understanding how activities affect humans and the environment. It links models that integrate across scientific disciplines, allowing for tailored solutions to specific activities, and it provides meaningful information to business and technical managers. FRAMES is the key to identifying, analyzing, and managing potential environmental, safety, and health risks. The purpose of FRAMES is to assist users in developing environmental

\footnotetext{
${ }^{1}$ http://mepas.pnl.gov/FramesV2/index.stm
} 
scenarios and to provide options for selecting the most appropriate computer codes to conduct human and environmental risk management analyses.

The uncertainty approach of Meyer et al. (2007) uses information resulting from a maximum likelihood or least squares model parameter estimation to provide comparative measures of model predictive performance. This report describes the integration within FRAMES of the parameter estimation software PEST $^{2}$ (Doherty 2004). The module implementing this integration will be referred to as F2PEST. PEST is model-independent software implementing a variety of parameter estimation methods, including nonlinear regression of a generalized least squares objective function, from which the model comparison statistics are computed. During execution PEST will iteratively try different values for a model's input parameters and then compare a selected set of simulated model outputs to a given set of expected outputs, typically observed (measured) values, using the results of this comparison to compute new input parameter values.

While the complete documentation for the file formats PEST uses is available in the PEST documentation, a summary is provided here as context for the design. PEST interfaces to a model through three file types. The first file type that PEST uses is an input file template. This is a file that describes an input file (or data file) that needs to be modified by PEST, typically a model input file containing model parameters. The template file uses a mark-up approach to note where important values are and assigns aliases to those values. Associated with the input file template is the model input file that will be read. Table 1.1 is an example of an input file and associated template file.

Table 1-1. Example model input file and corresponding PEST template file

\begin{tabular}{|ll|lll|}
\hline \multicolumn{2}{|c|}{ Example Model Input (Data) File } & \multicolumn{3}{c|}{ Corresponding Input File Template } \\
\hline Model input & ptf \# & & \\
12 & Number of months & Model input & \\
40 & Number of years & \#NMonths $\#$ & Number of months \\
65 & Concentration & \#NYears $\quad \#$ & Number of years \\
& & \#Conc $\quad \#$ & Concentration \\
\hline
\end{tabular}

The lines on the left of Table 1.1 represent the original input file including comments and descriptive information. The lines on the right represent the associated template file that contains the aliases NMonths, NYears, and Conc that should be associated with the values of 12, 40, and 65 respectively.

The second type of file PEST uses is an instruction file that describes the location of selected model output values and assigns alias names. Because reading an output file requires certain values to be found at locations marked by text labels, PEST allows for commands to be provided

${ }^{2}$ http://www.sspa.com/pest/ 
specifying how to read the data that follows. In the F2PEST tools the format is very simple because the F2PEST module writes the model output. In this case, the PEST "11" command ("ell1 ") is sufficient to read the corresponding output from a FRAMES model system. Each line of the instruction file illustrated in Table 1-2 tells PEST to advance one line in the model output file and read the output value between columns 1 and 7 for the observation named in parentheses, e.g., c3.

Table 1-2. Example model output file and corresponding PEST instruction file

\begin{tabular}{|l|l|}
\hline \multicolumn{1}{|c|}{ Example Model Output File } & \multicolumn{1}{c|}{ Corresponding Instruction File } \\
\hline 0.11948 & pif \# \\
0.29620 & $I 1(c 1) 1: 7$ \\
0.50162 & $I 1(c 2) 1: 7$ \\
0.67325 & $I 1(c 3) 1: 7$ \\
& $I 1(c 4) 1: 7$ \\
\hline
\end{tabular}

The third file used by PEST is the control file, which is given an extension of .pst. This file defines the methods used by PEST in the parameter estimation, contains the initial and allowed values for the model input parameters, and contains the observed (or measured) outputs and weights. 


\subsection{Requirements}

Requirements are characteristics and behaviors that a piece of software must possess to function adequately for its intended purpose. The Frames 2.0 Pest Integration (F2PEST) represents the ability in FRAMES 2.0 to perform a parameter estimation analysis across a set of environmental models for a single site. The F2PEST will be constructed in such a way that allows for multiple invocations of the PEST code within a single FRAMES 2.0 conceptual site model.

The F2PEST is a module that allows PEST to operate on a subset of FRAMES 2.0 modules that are linked together in the FRAMES conceptual site model diagram. Figure 2-1 shows a diagram and the F2PEST as part of that diagram. The environmental models (A1 and B1) shown could be Groundwater Modeling System ${ }^{3}$ or other FRAMES 2.0 compatible environmental models. From a diagrammatic standpoint, PEST manipulates models A1 and B1 separately which means that separate PEST templates, instructions, and control files exist for both instances. The Multi-model Summarizer module shown in Figure 2-1 represents a capability to use the parameter estimation results (and other outputs from the models or uncertainty modules) to compute model comparison statistics and multi-model predictions.

The F2PEST will consume a number of modules in a way that provides inputs and outputs from the module. This means that the F2PEST needs to run as a system processor.

The main requirements of the F2PEST are:

1. Run as part of a FRAMES 2.0 simulation.

2. Consume any number of module outputs and inputs from models connected to the F2PEST module. These are the list of modules that will have their results analyzed and inputs manipulated.

3. Produce outputs that include the final objective function value, parameter estimates, the parameter estimation covariance matrix, observed values and weights, simulated values, residuals, standard error variance, and the model selection criteria (as computed by PEST $)^{4}$.

4. Accept the arguments of FRAMES 2.0 path, simulation name, and module name when invoked.

5. Run as a system level component of FRAMES 2.0

6. Have a user interface that gathers the inputs and outputs to be processed in a way that

\section{${ }^{3}$ http://chl.erdc.usace.army.mil/gms}

${ }^{4}$ The F2PEST code implemented reads additional PEST output: observed values and weights, simulated values, residuals, and the standard error variance. These are not currently produced as output by F2PEST. 
allows a user to select any input or output value from any model that feeds data to the F2PEST module. This is similar to the behavior of the FRAMES Sensitivity and Uncertainty Module, which allows the user to choose a subset of inputs and outputs from all the connected models.

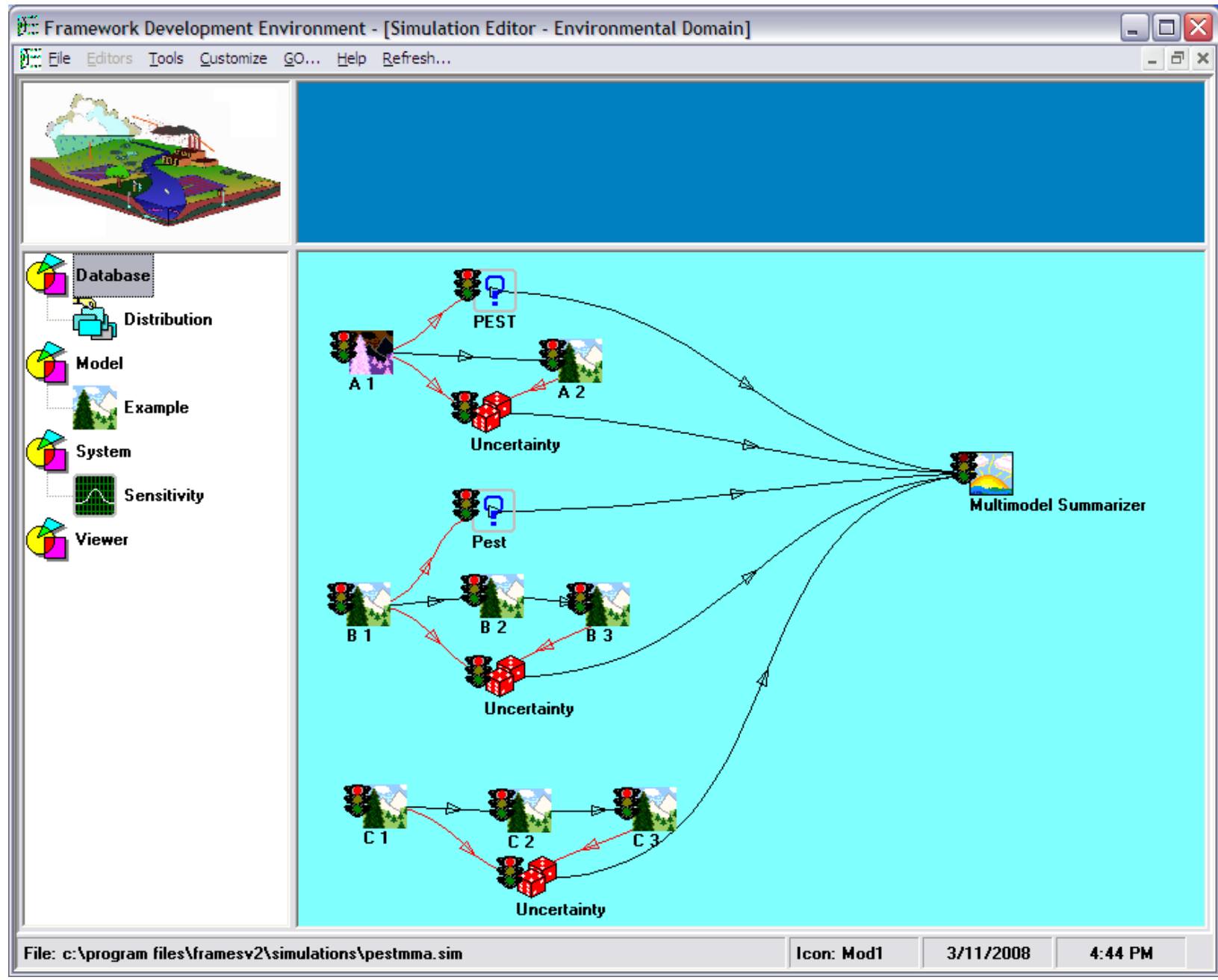

Figure 2-1. An example FRAMES 2.0 diagram that includes F2PEST modules 


\subsection{Design}

Design elements are strategies for meeting requirements. The F2PEST is designed to meet the requirements identified in Section 2.0. Key to meeting those requirements is the interface between the FRAMES 2.0 datasets and PEST. The design for F2PEST is staged and straightforward. In the first stage the focus is to have PEST execute on a series of FRAMES modules. In Figure 2-1 PEST is shown to only connect to one module, but in general it needs to be able to connect to any number of modules. The effort put in to executing one model is just the same as for many because FRAMES 2.0 has a Run Between function that allows a system processor to execute all modules between two modules. If a single module is desired then Run Between is give the same module identifier for both the start and the end.

When the F2PEST interface is run, the user is prompted for the User Interface datasets and associated variables that need to be manipulated for the calibration. The user is also prompted for the Boundary Condition datasets (usually the result of computation) and associated variables that represent the observed values and model outputs to be used in the parameter estimation. Examples of the user interface screens for input of estimated parameters and observed values are shown in Figure 5-3 and Figure 5-4. Lastly a PEST control file is created that is initially populated for all the inputs and the observations when the user switches to the tab showing this information. An example of this screen is shown in Figure 5-5. With this approach, expert PEST users will find it very convenient to modify the control file (using a standard text editor) to have PEST perform exactly as they wish. A more novice PEST user can accept the default control parameters. For future development, a helpful application called a "Wizard" could be written that asks clear questions of the novice user and then generates a PEST control file with exactly what they need.

To implement F2PEST, three executables need to be created. The first is the F2PEST User Interface described above. The second is the PEST Pre/Post processor that runs PEST with the created PEST control file. This application reads the FRAMES 2.0 datasets and writes the PEST template file and an example text file with current values in appropriate locations. A PEST instruction file is also generated along with an example output file. So from PEST's view it is invoked with a template file, instruction file, and control file. When PEST invokes the "model" the last executable is invoked.

The last executable takes the input file, which has been updated by PEST, and updates the FRAMES 2.0 datasets. It then executes Run Between on all modules connected to the PEST icon. When Run Between completes successfully the output datasets are read and written to the output file, which is then read by PEST. Figure 3-1 is a block diagram of F2PEST integration components.

The location of PEST is assumed to be given in the PEST_PATH environment variable. All PEST files are written to the location specified in PEST_PATH. 


\section{FRAMES PEST UI (RunF2PestUI. bat)}

Define subset of input dataset variables that will be in ModX.tpl/.dat Define subset of boundary condition variables that will be in ModX.out/.ins Define additional commands for PEST via a notepad type interface

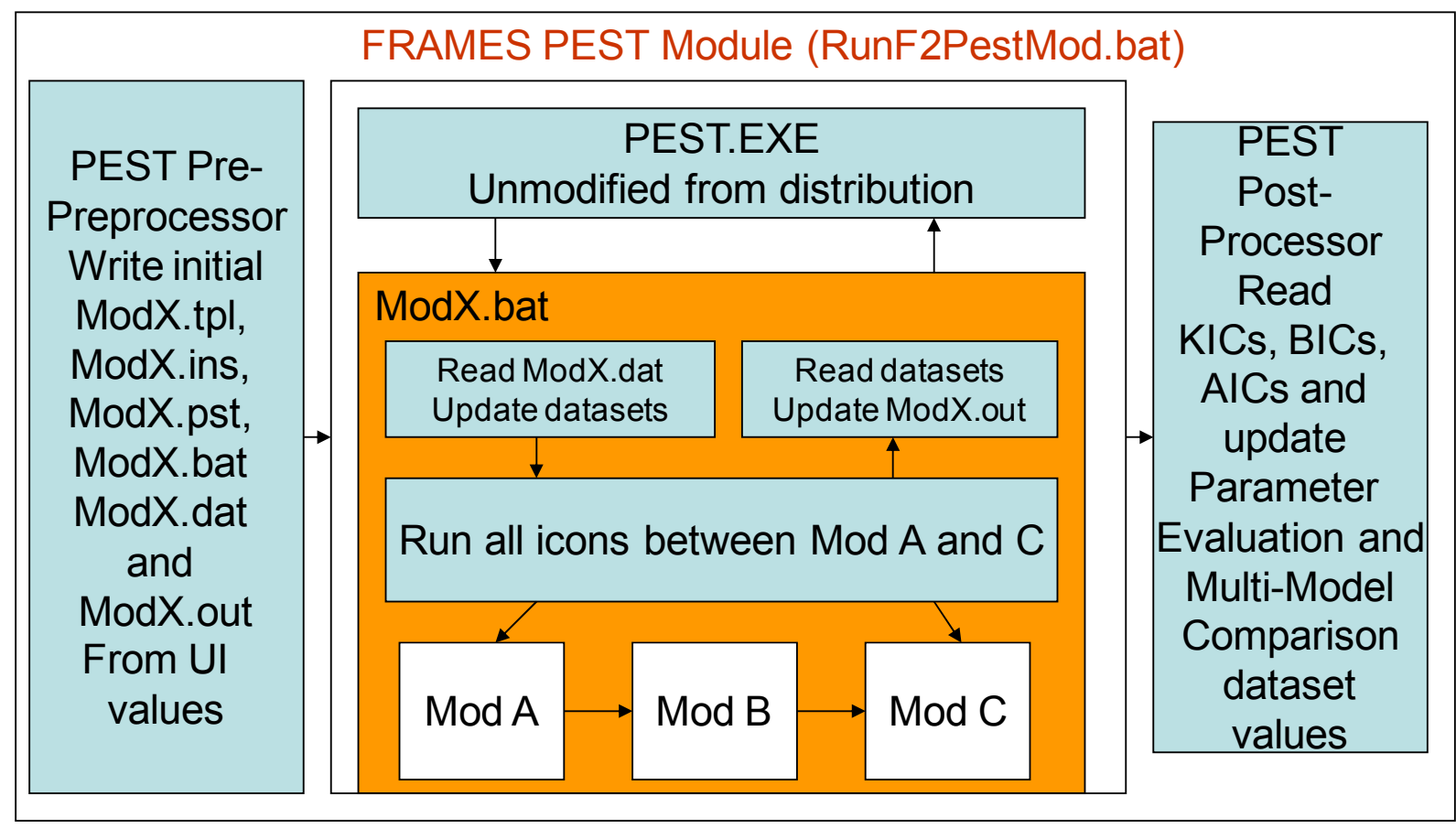

Figure 3-1. F2PEST integration diagram

\subsection{Limitations and Issues}

F2PEST is built on top of the PEST executables 11.5. Any limitations in capability are expected limitations of F2PEST.

F2PEST in its initial phase of implementation does not intend to build a complete PEST user interface for PEST. Instead it intends to use defaults (i.e., recommended values in the PEST documentation) for some of the control parameters. 


\subsection{Specifications}

Specifications are the descriptions of input and output file that are used during execution of a component. Alongside these input and output descriptions how the component is invoked and how the component was compiled are also discussed. If a virtual environment like Java is used for execution, the version of that environment is also a specification.

The F2PEST module reads any data set that is a module input or boundary condition used by subordinate modules. The data (.dat) and template (.tpl) files generated by F2PEST (discussed in the Introduction) have a simple format. Every alias specified in the user interface will be written to a line of the data file. The template file will contain the appropriate pound sign (\#) framing described in the PEST documentation with the alias between the pound signs. There are always 12 character positions allocated for writing values to the data file. The data file is manipulated by PEST, the template file is read by PEST. See Table 1-1 for an example of a data file and template file pair. Note: that comments are not currently generated by F2PEST.

The formats used in the instruction (.ins) and the output (.out) file are similar to the data and template file. The output file contains one observed value on each line of the file. The instruction file also contains one line for every observed value in the output file and contains the starting 'pif' command. Table 1-2 is an example of the instruction and the output file pair. These files are read by PEST and follow format specified by PEST.

The index (idx1, idx2, .. idxN) columns seen in the F2PEST user interface (e.g., Figure 5-3 and Figure 5-4) allow a user to specify which subset of values are to be used in an analysis or manipulated. For parameters, the indices specify what values will be manipulated in a dataset. In the current implementation only a single value should be specified for a parameter. If a variable from a FRAMES 2.0 dataset that contains more than one dimension is specified as a parameter to be estimated, all indices should be specified with a single integer value to define which value in the collection of values is to be the parameter. If two values in a single variable need to be manipulated (i.e., a single variable but two different index specifications), two aliases are needed and the different indices for the two parameters need to be provided by the user. Similar logic is expected for observed values. Each alias should represent only one model output value even if the FRAMES 2.0 dataset variable has more than one dimension. The user should specify a set of indices that identify the single model output value that is to be compared to an observed value. If a single variable with different indices is needed for comparison with multiple observed values, then multiple aliases should be added, one for each specific set of indices/observed value.

F2PEST writes the ParameterEvaluation and MultiModelComparison dictionaries. The ParameterEvaluation dictionary contains the results of the PEST parameter estimation, such as the optimal parameter estimates and the parameter estimation covariance matrix. The MultiModelComparison dictionary contains the model comparison statistics computed by PEST. The contents and characteristics of the dictionaries produced by F2PEST are given in Table 4-1.

As noted above, the location of PEST is assumed to be given in the PEST_PATH environment variable. All PEST files are written to the location specified in PEST_PATH. 


\subsection{Command Line}

The command line for the F2PEST is the standard FRAMES module command line. The module when invoked is passed the FRAMES 2.0 path, the Simulation Name, and the Module Name. These three pieces of information are required to connect to the FRAMES system with the SystemIO.dll. The FRAMES 2.0 path is assumed to be contained in the FRAMES_PATH environment variable.

\subsection{Compiler Version}

The user interface is compiled using Java. The project file is F2PESTUI.mak. The computational engine of the F2PEST is compiled using $\mathrm{c}++$ compiler and gcc compiler version 3.4 or better. The project file for the computation component is F2PEST.mak

\subsection{Java Runtime Environment Version}

Java 1.3 or better is required to run the user interface. 
Table 4-1. Dictionaries produced by F2PEST: ParameterEvaluation containing parameter estimation results and MultiModelComparison containing model comparison statistics

\begin{tabular}{|c|c|c|c|c|c|c|c|c|c|c|c|}
\hline Name & Description & Unit & Measure & Type & Range & S & D & U & $\mathbf{K}$ & Prep & Indices \\
\hline Alias & $\begin{array}{l}\text { Alias of the parameter } \\
\text { that is being reported }\end{array}$ & & & STRING & $0-80$ & $\mathrm{Y}$ & 1 & $\mathrm{~N}$ & $\mathrm{~N}$ & & \\
\hline estimates & $\begin{array}{l}\text { Estimate for the } \\
\text { parameter }\end{array}$ & & & FLOAT & $\begin{array}{l}-1 \mathrm{E}+38- \\
1 \mathrm{E}+38\end{array}$ & $\mathrm{~N}$ & 1 & $\mathrm{~N}$ & $\mathrm{~N}$ & & Alias \\
\hline Indices & $\begin{array}{l}\text { Indices of the } \\
\text { parameter being } \\
\text { evaluated }\end{array}$ & & & STRING & $0-80$ & $\mathrm{Y}$ & 2 & $\mathrm{~N}$ & $\mathrm{~N}$ & & Alias \\
\hline lowerLimit & $\begin{array}{l}\text { 95\% lower limit of } \\
\text { parameter estimate }\end{array}$ & & & FLOAT & $\begin{array}{l}-1 E+38- \\
1 E+38\end{array}$ & $\mathrm{~N}$ & 1 & $\mathrm{~N}$ & $\mathrm{~N}$ & & Alias \\
\hline parameterCorrelation & & & & FLOAT & $\begin{array}{l}-1 \mathrm{E}+38- \\
1 \mathrm{E}+38\end{array}$ & $\mathrm{~N}$ & 2 & $\mathrm{~N}$ & $\mathrm{~N}$ & & Alias \\
\hline parameterCovariance & & & & FLOAT & $\begin{array}{l}-1 E+38- \\
1 E+38\end{array}$ & $\mathrm{~N}$ & 2 & $\mathrm{~N}$ & $\mathrm{~N}$ & & Alias \\
\hline Set & $\begin{array}{l}\text { Dataset associated } \\
\text { with the Alias }\end{array}$ & & & STRING & $0-80$ & $\mathrm{~N}$ & 1 & $\mathrm{~N}$ & $\mathrm{~N}$ & & Alias \\
\hline Type & $\begin{array}{l}\text { Type of variables being } \\
\text { analyzed }\end{array}$ & & & STRING & $0-80$ & $\mathrm{~N}$ & 1 & $\mathrm{~N}$ & $\mathrm{~N}$ & & Alias \\
\hline Units & $\begin{array}{l}\text { Units of the variable } \\
\text { being analyzed }\end{array}$ & & & STRING & $0-80$ & $\mathrm{~N}$ & 1 & $\mathrm{~N}$ & $\mathrm{~N}$ & & Alias \\
\hline upperLimit & $\begin{array}{l}95 \% \text { confidence upper } \\
\text { limit of the parameter } \\
\text { values }\end{array}$ & & & FLOAT & $\begin{array}{l}-1 \mathrm{E}+38- \\
1 \mathrm{E}+38\end{array}$ & $\mathrm{~N}$ & 1 & $\mathrm{~N}$ & $\mathrm{~N}$ & & Alias \\
\hline Variables & $\begin{array}{l}\text { Variable names for the } \\
\text { parameter being } \\
\text { analyzed }\end{array}$ & & & STRING & $0-80$ & $\mathrm{~N}$ & 1 & $\mathrm{~N}$ & $\mathrm{~N}$ & & Alias \\
\hline
\end{tabular}

MultiModelComparison

\begin{tabular}{|c|c|c|c|c|c|c|c|c|c|c|c|}
\hline Name & Description & Unit & Measure & Type & Range & $\mathbf{s}$ & D & $\mathbf{U}$ & $\mathbf{K}$ & Prep & Indices \\
\hline $\mathrm{AIC}$ & $\begin{array}{l}\text { Akaike Information } \\
\text { Criterion }\end{array}$ & & & FLOAT & $\begin{array}{l}-1 \mathrm{E}+38- \\
1 \mathrm{E}+38\end{array}$ & $\mathrm{~N}$ & 0 & $\mathrm{~N}$ & $\mathrm{~N}$ & & \\
\hline $\mathrm{AICC}$ & $\begin{array}{l}\text { Modified Akaike } \\
\text { Information Criterion }\end{array}$ & & & FLOAT & $\begin{array}{l}-1 \mathrm{E}+38- \\
1 \mathrm{E}+38\end{array}$ & $\mathrm{~N}$ & 0 & $\mathrm{~N}$ & $\mathrm{~N}$ & & \\
\hline AICCNotComputed & $\begin{array}{l}\text { Modified Akaike } \\
\text { Information Criterion } \\
\text { available }\end{array}$ & & & LOGICAL & $0-1$ & $\mathrm{~N}$ & 0 & $\mathrm{~N}$ & $\mathrm{~N}$ & & \\
\hline 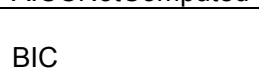 & $\begin{array}{l}\text { Bayesian Information } \\
\text { Criterion }\end{array}$ & & & FLOAT & $\begin{array}{l}-1 \mathrm{E}+38- \\
1 \mathrm{E}+38\end{array}$ & $\mathrm{~N}$ & 0 & $\mathrm{~N}$ & $\mathrm{~N}$ & & \\
\hline $\mathrm{KIC}$ & $\begin{array}{l}\text { Kashyap Information } \\
\text { Criterion }\end{array}$ & & & FLOAT & $\begin{array}{l}-1 \mathrm{E}+38- \\
1 \mathrm{E}+38\end{array}$ & $\mathrm{~N}$ & 0 & $\mathrm{~N}$ & $\mathrm{~N}$ & & \\
\hline
\end{tabular}

Legend

\begin{tabular}{|l|l|}
\hline Column Name & Meaning \\
\hline S & Self-Indexed \\
\hline D & Dimensional Size \\
\hline U & Uncertainty can apply (Stochastic) \\
\hline K & Is the variable a key to others \\
\hline
\end{tabular}




\subsection{Testing Approach and Results}

A test case was developed to test the performance of the F2PEST module integration with FRAMES. The test case uses a model of groundwater transport with observations derived from the true model output with the addition of a random error term. F2PEST was used with the observation values to estimate the parameters of the model.

The one-dimensional advection-dispersion equation for transport of a conservative solute in a uniform, steady flow field can be written as

$$
D_{l} \frac{\partial^{2} C}{\partial l^{2}}-v \frac{\partial C}{\partial l}=\frac{\partial C}{\partial t}
$$

where $C$ is the solute concentration, $D_{l}$ is the dispersion coefficient along $l$, the direction of flow, $v$ is the groundwater velocity, and $t$ is time. For a semi-infinite domain with an initial concentration of zero and a step function source of $C=C_{0}$ at $l=0$, the solution to Eq. 1 can be written as (Freeze and Cherry 1979)

$$
\frac{C}{C_{0}}=\frac{1}{2}\left[\operatorname{erfc}\left(\frac{l-v t}{2 \sqrt{D_{l} t}}\right)+\exp \left(\frac{v l}{D_{l}}\right) \operatorname{erfc}\left(\frac{l+v t}{2 \sqrt{D_{l} t}}\right)\right]
$$

where $\operatorname{erfc}$ is the complementary error function.

Eq. 2 was solved for concentration as a function of time at a distance of $l=0.1$, using a source concentration of $C_{0}=1.0$ and parameters $v=1.0 \times 10^{-2}$ and $D_{l}=5.0 \times 10^{-4}$ (all quantities with arbitrary consistent units). Concentration observations for use in F2PEST were based on the solutions to Eq. 2 at times of 3, 5, 8, 12,15, 17, and 20. Each concentration at these times was perturbed by an amount randomly sampled from a normal distribution with a mean of 0.0 and a standard deviation equal to 20 percent of the concentration. Calculated solution concentrations and randomly perturbed observed values are listed in Table 5-1. A plot of the calculated solution along with the perturbed observations is given as Figure 5-1. Note that the observed values are biased low compared to the calculated solution from which they were derived.

A FRAMES module implementing Eq. 2 as a model was written for the test case. For this test, the observations from Table 5-1 were used. $C_{0}$ was assumed to be known, while $v$ and $D_{l}$ were fit to the observations using F2PEST. The initial values used were $v=2.0 \times 10^{-2}$ and $D_{l}=1.0 \times 10^{-4}$. The F2PEST parameter estimation module was connected to the model in the FRAMES simulation editor, as shown in Figure 5-2. The F2PEST user interface contains three separate tabs, one for selecting model parameters and entering parameter information (such as initial values, upper and lower bounds, and transformations), one for selecting model output variables and entering observation values and weights, and a third tab for the PEST control file. The highlighted portion of the control file is not editable from the F2PEST UI. All control parameters are available for editing using a text editor on the PEST control file itself, however. The three tabs of the F2PEST UI are shown in Figure 5-3, Figure 5-4, and Figure 5-5. 
Table 5-1. Solution concentrations from Eq. 2 and randomly perturbed observations for use in F2PEST

\begin{tabular}{|r|r|r|}
\hline Time & $\begin{array}{c}\text { Solution } \\
\text { Concentration }\end{array}$ & $\begin{array}{c}\text { Perturbed } \\
\text { Observation }\end{array}$ \\
\hline 3 & 0.165727 & 0.071169 \\
\hline 5 & 0.364976 & 0.367855 \\
\hline 8 & 0.574724 & 0.427322 \\
\hline 12 & 0.737246 & 0.706692 \\
\hline 15 & 0.810768 & 0.777205 \\
\hline 17 & 0.846108 & 0.870955 \\
\hline 20 & 0.885475 & 0.739659 \\
\hline
\end{tabular}

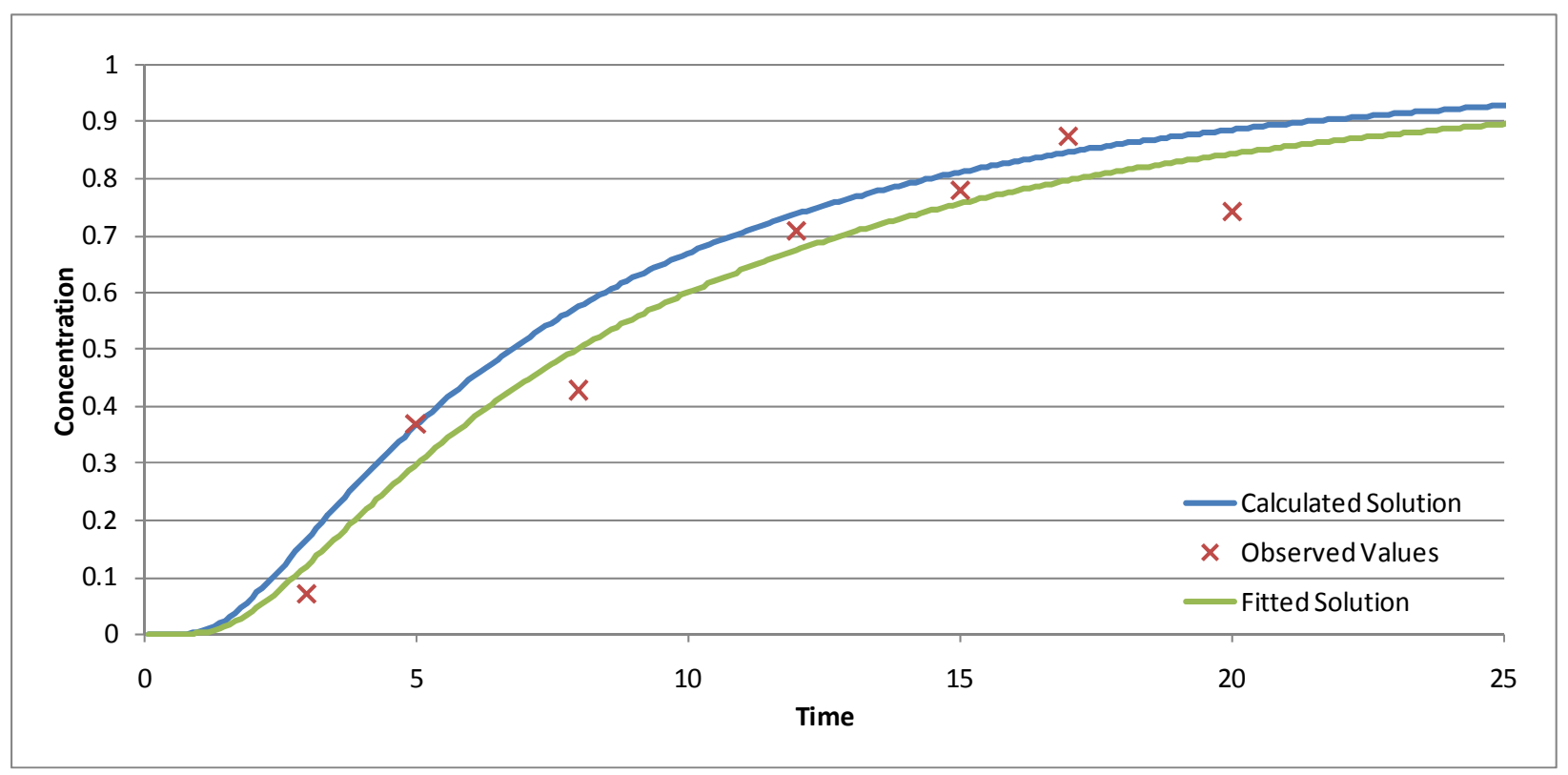

Figure 5-1. Calculated solution to Eq. 2 and randomly perturbed observation values used in F2PEST.

Execution of F2PEST for the test case produced the PEST .tpl, .ins, .dat, and .out files. The .dat file is the model input file containing the current iteration's values of the model parameters being estimated. The .tpl file is the template file instructing PEST on how to read and write to the dat file. The .out file is the model output file containing the current iteration's values of the modelsimulated observations. The ins file instructs PEST on how to read the out file. The final state of the files is shown in Table 5-2. The PEST history of execution and resulting solution is shown in the PEST .rec file (Appendix A). Final estimated parameter values were $v=8.408 \times 10^{-3}$ and $D_{l}$ $=4.316 \times 10^{-4}$. The fitted concentration solution using these parameter values is shown in Figure $5-1$. 
The dictionaries produced by the F2PEST module for the test case are shown in Figure 5-6 through Figure 5-8. Values contained in the dictionaries are the same as those in the PEST rec file demonstrating the correct execution of F2PEST for the test case.

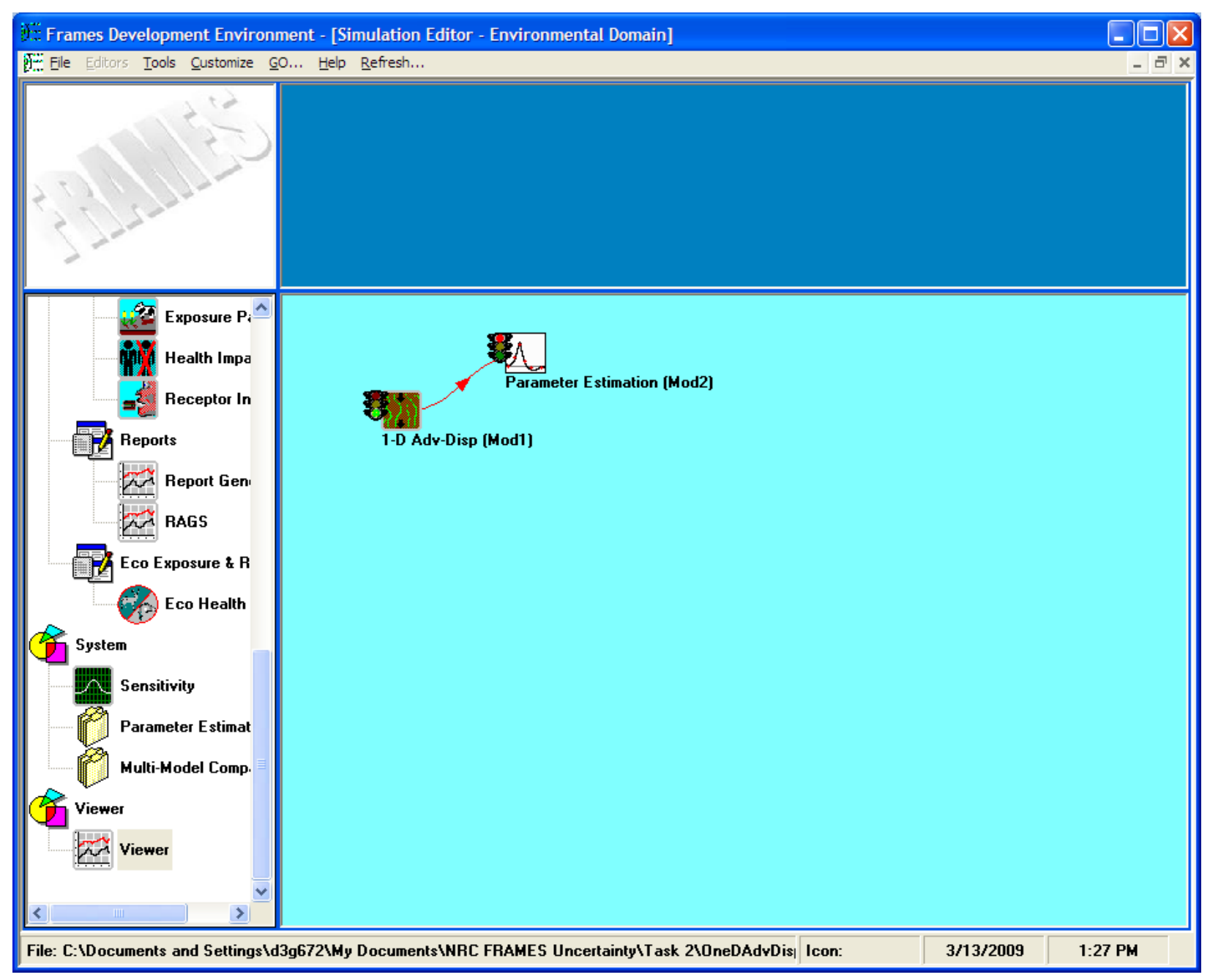

Figure 5-2. Simulation editor in FRAMES illustrating the F2PEST test case 


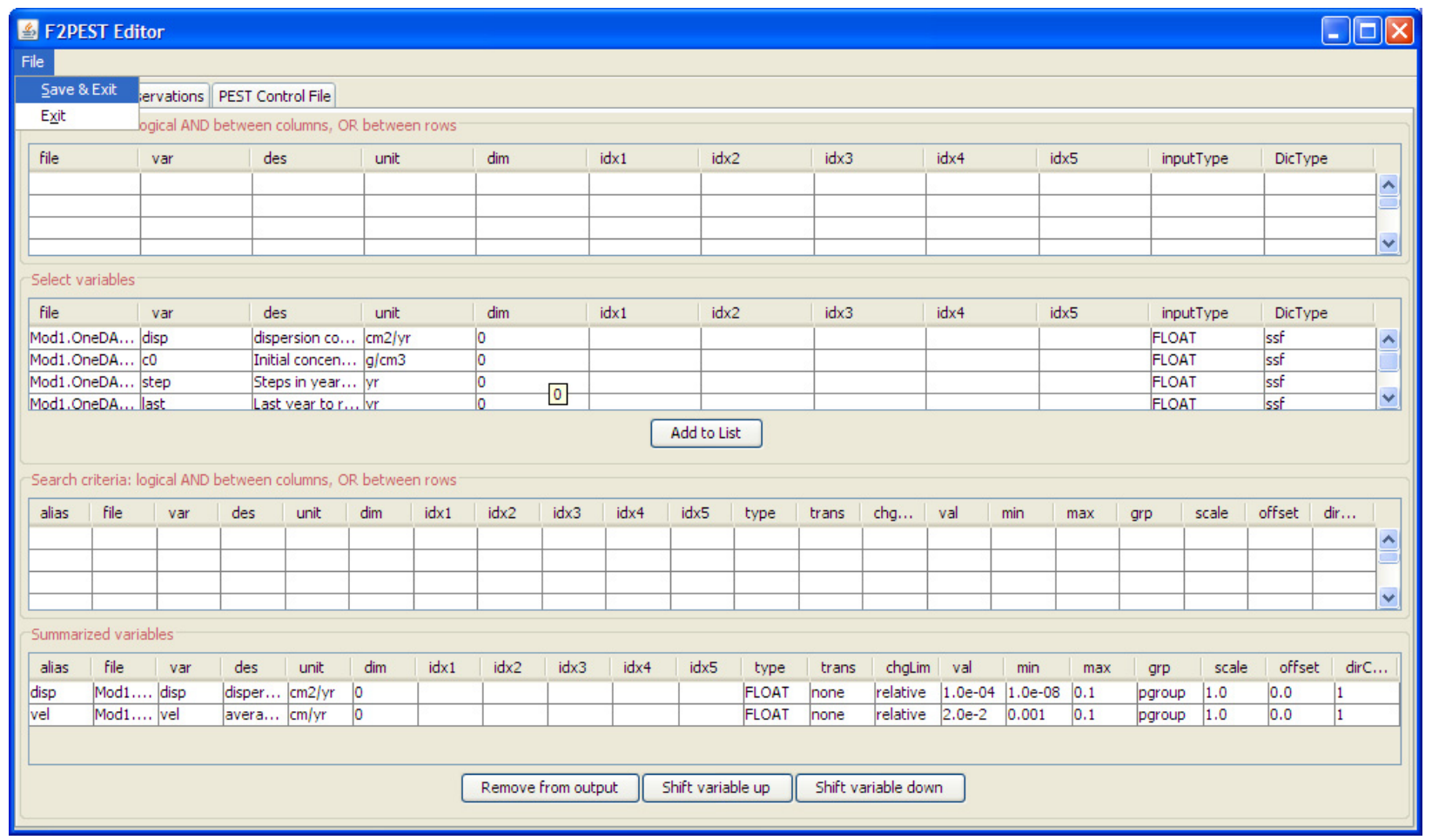

\section{Figure 5-3. Parameter selection tab from the F2PEST UI}

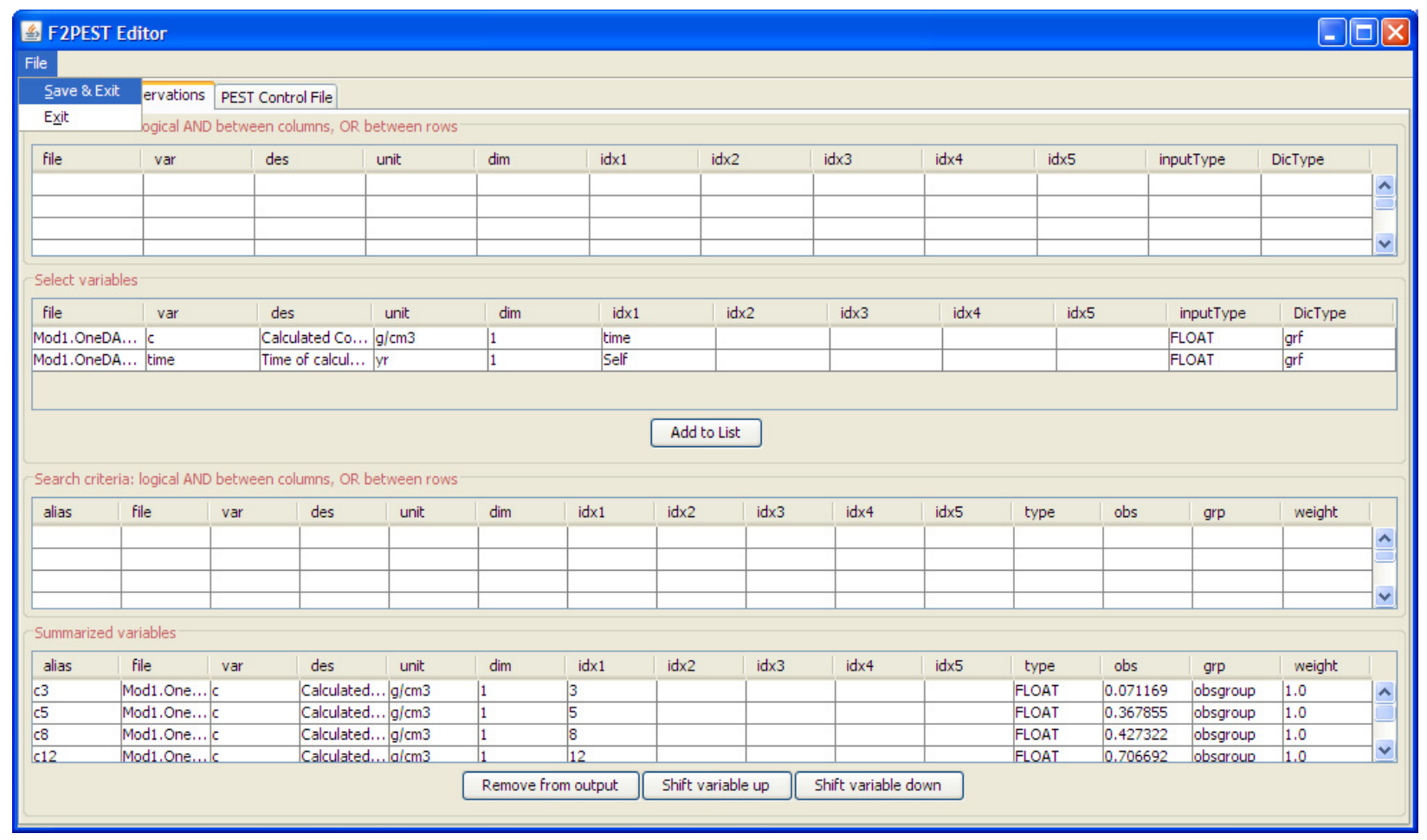

Figure 5-4. Observation selection tab from the F2PEST UI 


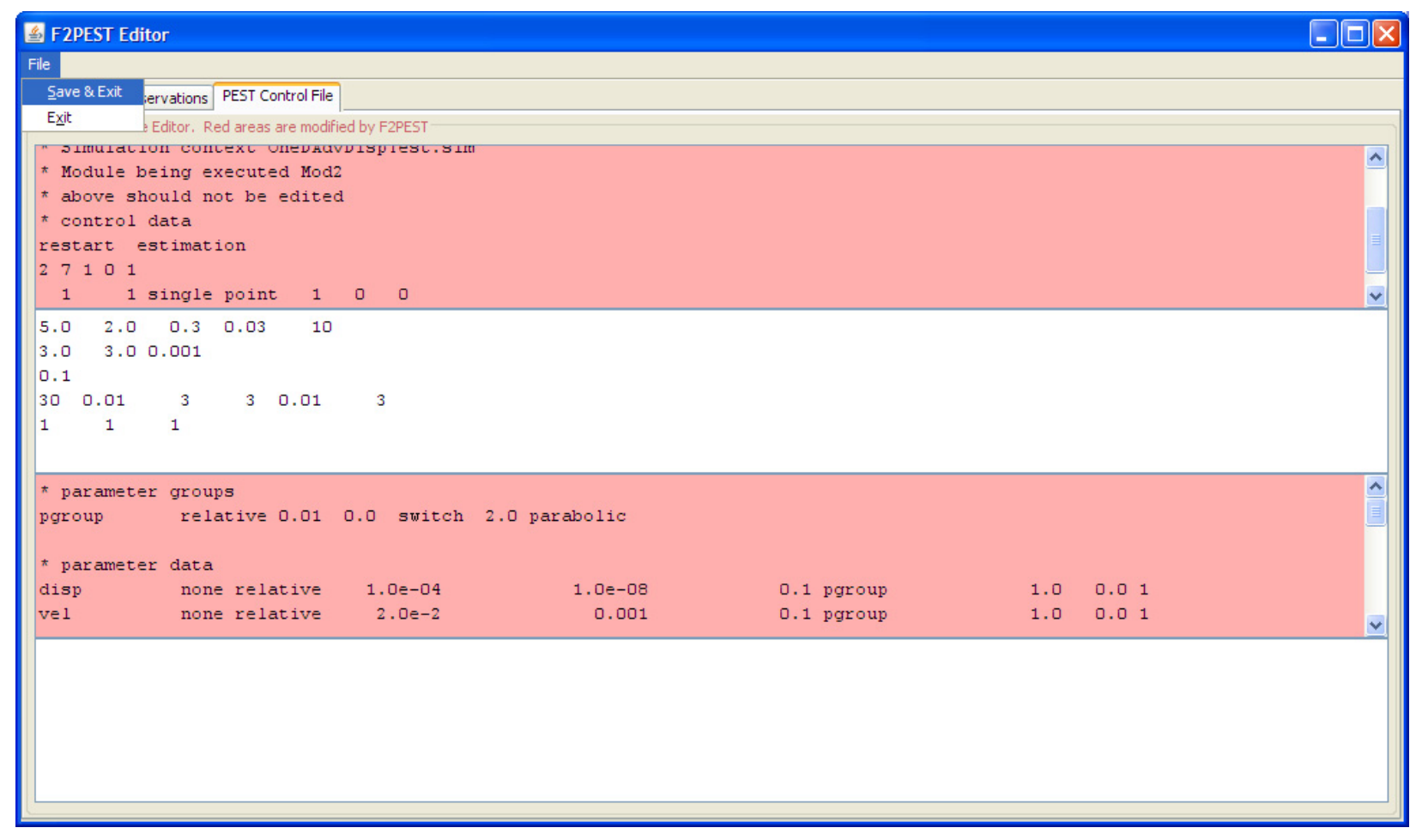

Figure 5-5. PEST control file tab from the F2PEST UI

Table 5-2. PEST files generated by F2PEST (final state)

\begin{tabular}{|ll|l|l|l|}
\hline .tpl & & .dat & .ins & .out \\
\hline ptf \# & & $4.3157554 \mathrm{E}-04$ & pif \# & 0.11948322 \\
$\#$ & disp \# & $8.4083119 \mathrm{E}-03$ & 11 (c3) $1: 12$ & 0.29619867 \\
$\#$ & vel \# & & 11 (c5) $1: 12$ & 0.50162905 \\
& & 11 (c8) $1: 12$ & 0.6732685 \\
& & 11 (c12) $1: 12$ & 0.75530654 \\
& & 11 (c15) $1: 12$ & 0.7960535 \\
& & 11 (c17) $1: 12$ & 0.8427709 \\
& & 11 (c20) $1: 12$ & \\
\hline
\end{tabular}




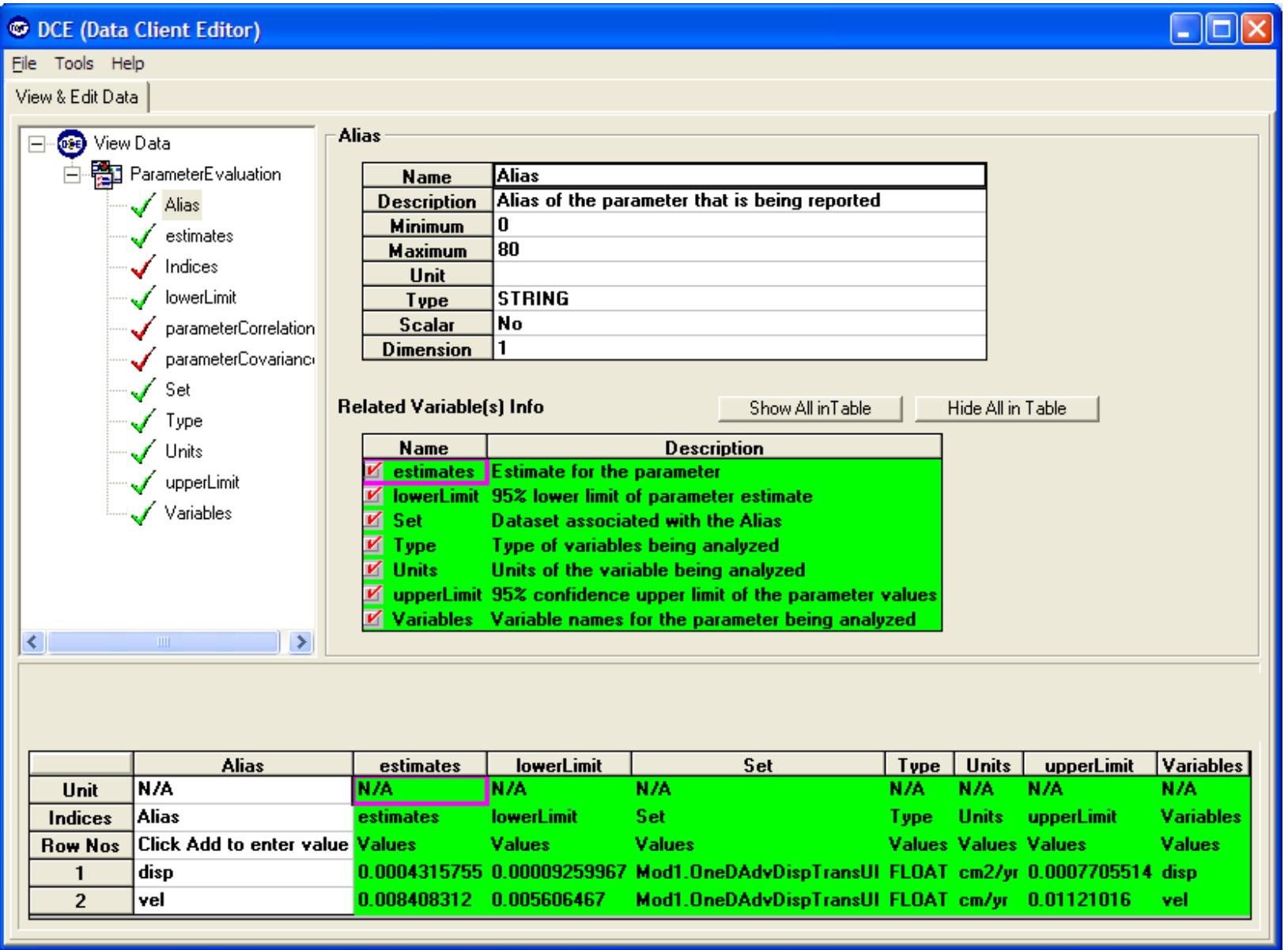

\section{Figure 5-6. ParameterEvaluation dictionary produced by F2PEST including parameter estimates and $95 \%$ confidence limits}




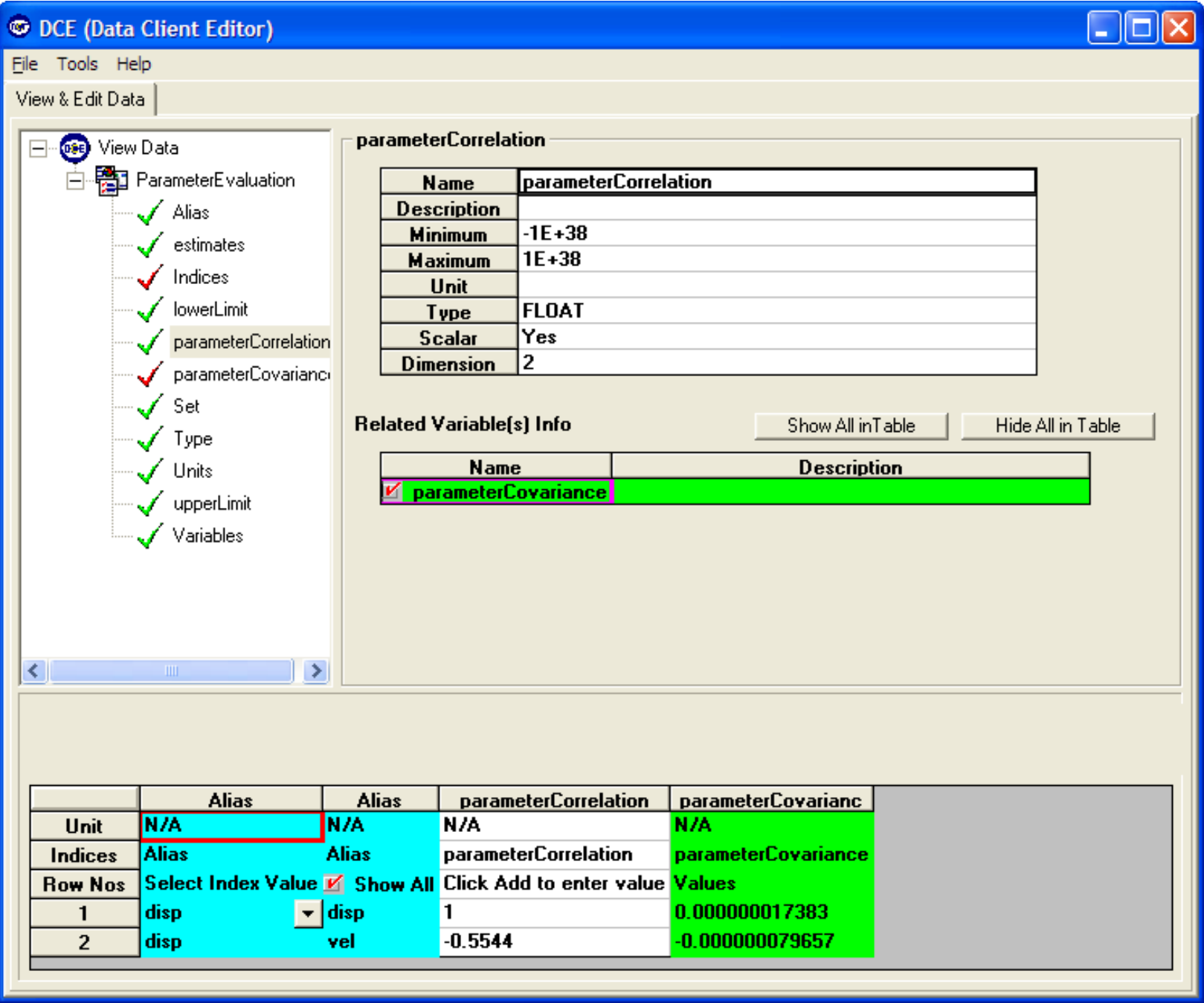

Figure 5-7. ParameterEvaluation dictionary produced by F2PEST including parameter covariance and correlation matrices 


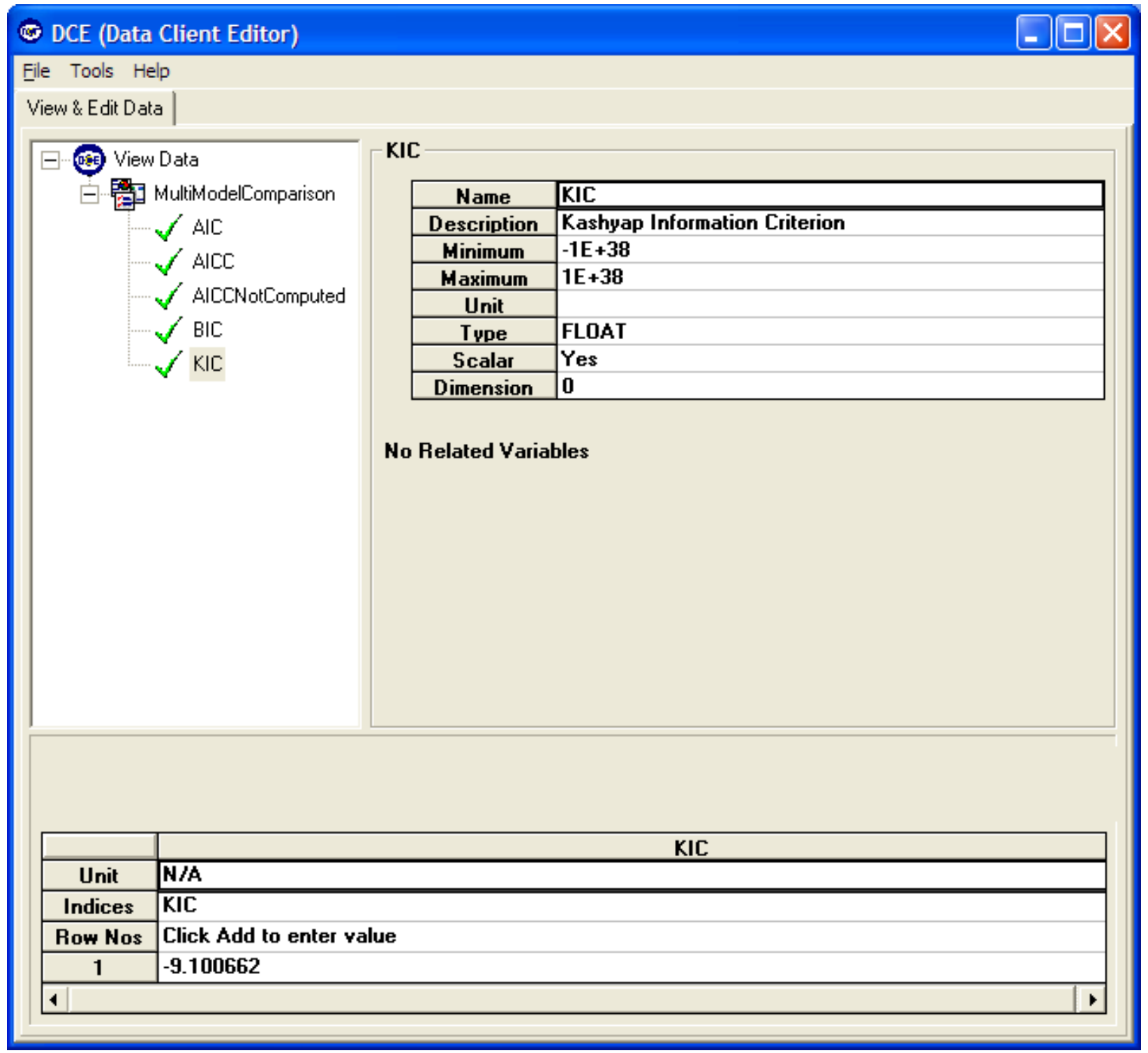

Figure 5-8. MultiModelComparison dictionary produced by F2PEST 


\subsection{References}

Doherty, J. 2004. PEST, Model-Independent Parameter Estimation, User's Manual: 5th Edition, Watermark Numerical Computing, Australia. http://www.sspa.com/pest/

Freeze, R.A. and J.A. Cherry. 1979. Groundwater, Prentice-Hall, Inc., Englewood Cliffs, New Jersey.

Meyer, P.D., M. Ye, M.L. Rockhold, S.P. Neuman, and K.J. Cantrell. 2007. Combined Estimation of Hydrogeologic Conceptual Model, Parameter, and Scenario Uncertainty with Application to Uranium Transport at the Hanford Site 300 Area, NUREG/CR-6940, U.S. Nuclear Regulatory Commission, Washington, DC. 
APPENDIX A

\section{PEST .rec File for Test Case}





\section{APPENDIX A \\ PEST .rec File for Test Case}

PEST RUN RECORD: CASE mod2

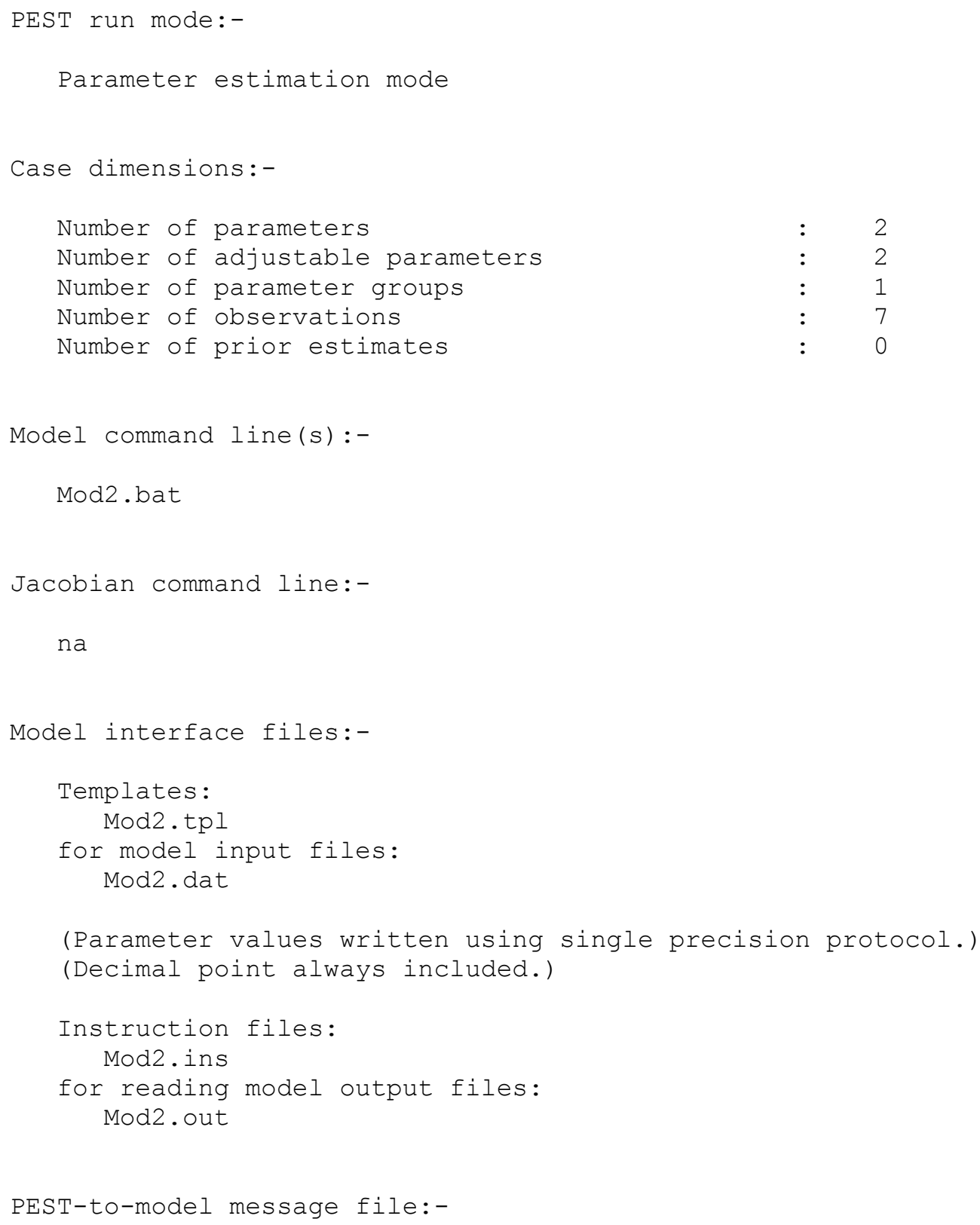




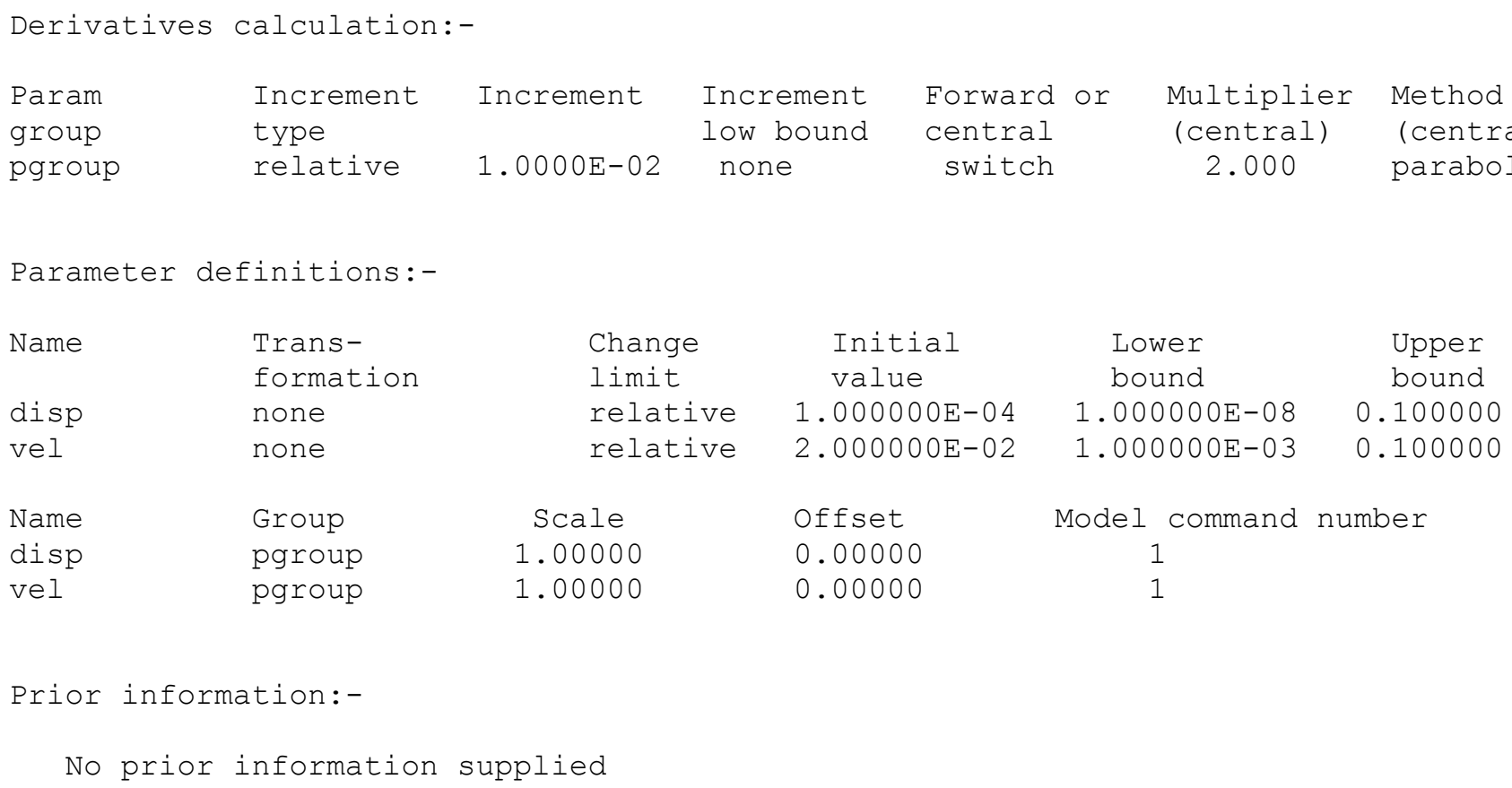

Observations:-

$\begin{array}{llll}\text { Observation name } & \text { Observation } & \text { Weight } & \text { Group } \\ \text { c3 } & 7.116900 \mathrm{E}-02 & 1.000 & \text { obsgroup } \\ \text { c5 } & 0.367855 & 1.000 & \text { obsgroup } \\ \text { c8 } & 0.427322 & 1.000 & \text { obsgroup } \\ \text { c12 } & 0.706692 & 1.000 & \text { obsgroup } \\ \text { c15 } & 0.777205 & 1.000 & \text { obsgroup } \\ \text { c17 } & 0.870955 & 1.000 & \text { obsgroup } \\ \text { c20 } & 0.739659 & 1.000 & \text { obsgroup }\end{array}$

Control settings:-

\begin{tabular}{|c|c|}
\hline Initial lambda & 5.0000 \\
\hline Lambda adjustment factor & 2.0000 \\
\hline Sufficient new/old phi ratio per optimisation iteration & 0.30000 \\
\hline Limiting relative phi reduction between lambdas & $3.00000 \mathrm{E}-02$ \\
\hline Maximum trial lambdas per iteration & 10 \\
\hline Perform Broyden's update of Jacobian matrix & no \\
\hline aximum factor parameter change (factor-limited changes) & : \\
\hline aximum relative parameter change (relative-limited changes) & 3.0000 \\
\hline $\begin{array}{l}\text { initial parameter values used in computing } \\
\text { t for near-zero parameters }\end{array}$ & $\mathrm{E}-03$ \\
\hline bending of parameter upgrade vector & no \\
\hline w parameters to stick to their bounds & no \\
\hline
\end{tabular}


Relative phi reduction below which to begin use of

Iteration at which to first consider derivatives switch

$: 0.10000$

Relative phi reduction indicating convergence

Number of phi values required within this range

Maximum number of consecutive failures to lower phi

Minimal relative parameter change indicating convergence

Number of consecutive iterations with minimal param change

Maximum number of optimisation iterations

$: 1$

Attempt automatic user intervention

$: 0.10000 \mathrm{E}-01$

$: 3$

$: 3$

$: 0.10000 \mathrm{E}-01$

$: 3$

$: \quad 30$

Attempt reuse of parameter sensitivities

: no

: no

File saving options: -

Save multiple JCO files

: no

Save multiple REI files

: no

OPTIMISATION RECORD

INITIAL CONDITIONS:

Sum of squared weighted residuals (ie phi) $=0.53293$

Current parameter values

$\begin{array}{ll}\text { disp } & 1.000000 \mathrm{E}-04 \\ \text { vel } & 2.000000 \mathrm{E}-02\end{array}$

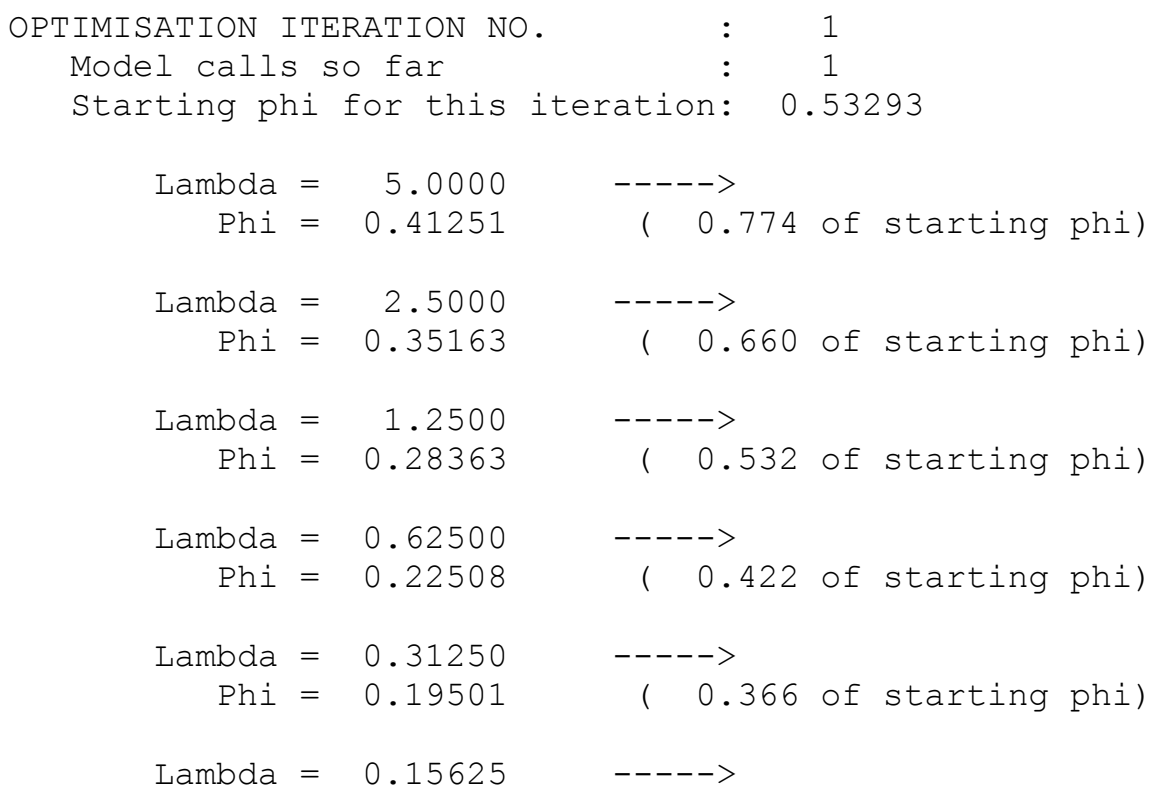




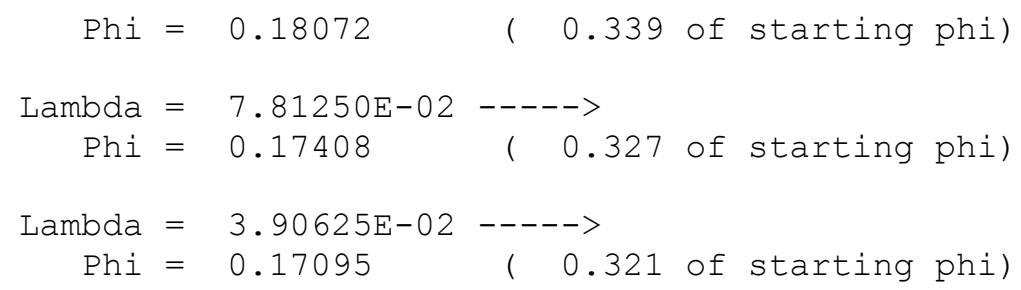

No more lambdas: relative phi reduction between lambdas less than 0.0300 Lowest phi this iteration: 0.17095

$\begin{array}{lrlr}\text { Current parameter values } & \text { Previous parameter values } \\ \text { disp } & 3.575853 \mathrm{E}-04 & \text { disp } & 1.000000 \mathrm{E}-04 \\ \text { vel } & 1.366885 \mathrm{E}-02 & \text { vel } & 2.000000 \mathrm{E}-02\end{array}$

Maximum relative change: 2.576 ["disp"]

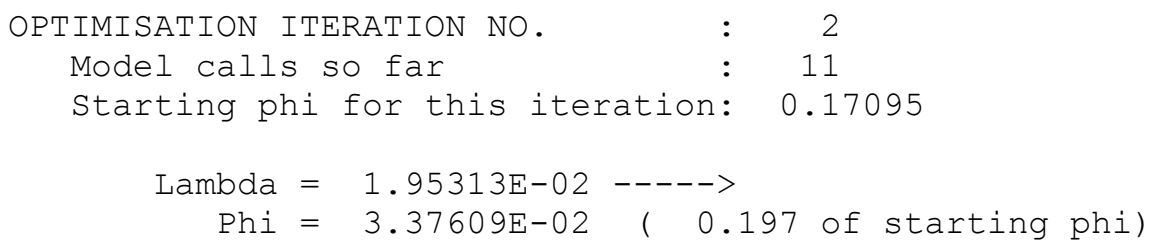

No more lambdas: phi is less than 0.3000 of starting phi Lowest phi this iteration: $3.37609 \mathrm{E}-02$

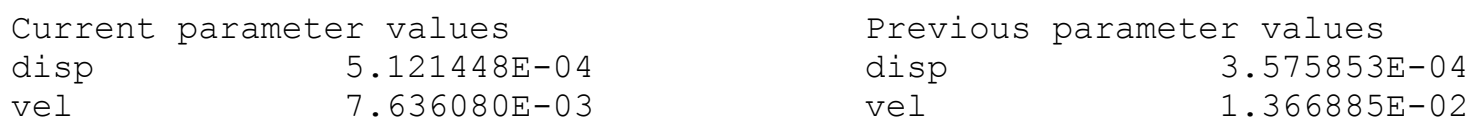

No more lambdas: relative phi reduction between lambdas less than 0.0300 Lowest phi this iteration: 3.08624E-02

Relative phi reduction between optimisation iterations less than 0.1000 Switch to central derivatives calculation

Current parameter values

disp $\quad 4.223738 \mathrm{E}-04$

vel $8.466615 \mathrm{E}-03$

Maximum relative change: 0.1753
Previous parameter values
disp
vel
vel $121448 \mathrm{E}-04$
$7.636080 \mathrm{E}-03$ 
Model calls so far : 18

Starting phi for this iteration: 3.08624E-02

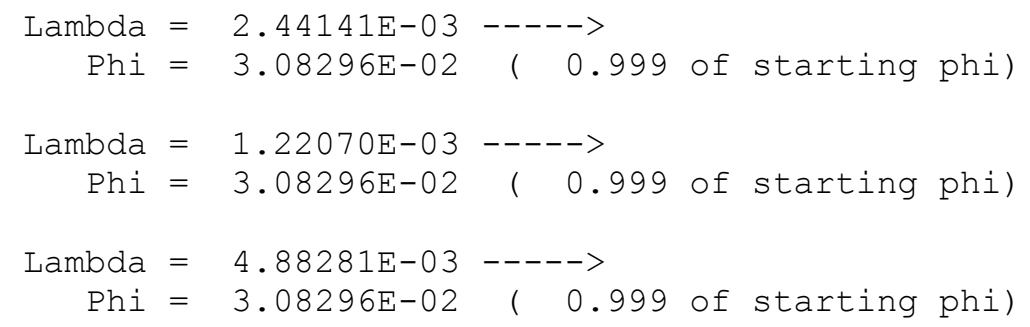

No more lambdas: phi rising

Lowest phi this iteration: 3.08296E-02

Current parameter values Previous parameter values

$\begin{array}{llll}\text { disp } & 4.309279 \mathrm{E}-04 & \text { disp } & 4.223738 \mathrm{E}-04 \\ \text { vel } & 8.414433 \mathrm{E}-03 & \text { vel } & 8.466615 \mathrm{E}-03\end{array}$

Maximum relative change: 2.0252E-02 ["disp"]

OPTIMISATION ITERATION NO. $\quad: \quad 5$

Model calls so far : 25

Starting phi for this iteration: 3.08296E-02

Lambda $=2.44141 \mathrm{E}-03----->$

$\mathrm{Phi}=3.08294 \mathrm{E}-02 \quad(1.000$ of starting phi $)$

Lambda $=1.22070 \mathrm{E}-03---->$

Phi $=3.08294 \mathrm{E}-02 \quad(1.000$ of starting phi $)$

Lambda $=4.88281 \mathrm{E}-03----->$

$\mathrm{Phi}=3.08294 \mathrm{E}-02 \quad(1.000$ of starting phi $)$

No more lambdas: phi rising

Lowest phi this iteration: $3.08294 \mathrm{E}-02$

Current parameter values Previous parameter values

$\begin{array}{lll}\text { disp } & 4.315755 \mathrm{E}-04 & \text { disp }\end{array}$

vel 8.408312E-03 vel 8.414433E-03

Maximum relative change: $1.5028 \mathrm{E}-03$ ["disp"]

Optimisation complete: the 3 lowest phi's are within a relative distance

of eachother of 1.000E-02

Total model calls: 32

The model has been run one final time using best parameters.

Thus all model input files contain best parameter values, and model

output files contain model results based on these parameters.

\author{
OPTIMISATION RESULTS
}

Parameters ----->

\title{
A.5
}




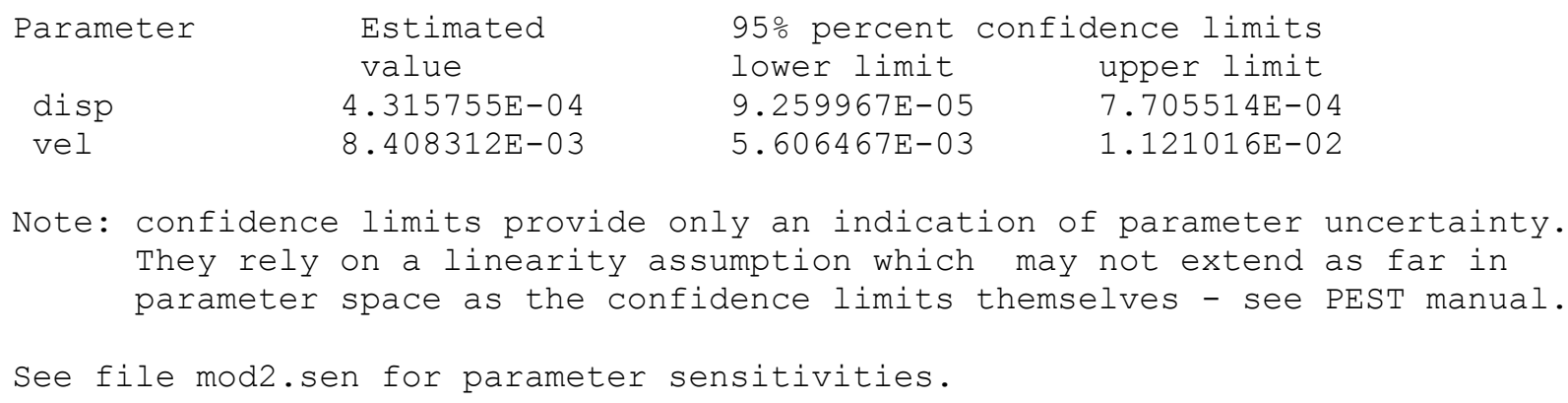

Note: confidence limits provide only an indication of parameter uncertainty. They rely on a linearity assumption which may not extend as far in parameter space as the confidence limits themselves - see PEST manual.

See file mod2.sen for parameter sensitivities.

\begin{tabular}{|c|c|c|c|c|c|}
\hline c5 & 0.367855 & 0.296199 & $7.165633 \mathrm{E}-02$ & 1.000 & obsgroup \\
\hline c8 & 0.427322 & 0.501629 & $-7.430705 E-02$ & 1.000 & obsgroup \\
\hline c12 & 0.706692 & 0.673269 & $3.342350 E-02$ & 1.000 & obsgroup \\
\hline c17 & 0.870955 & 0.796054 & $7.490150 \mathrm{E}-02$ & 1.000 & obsgroup \\
\hline c20 & 0.739659 & 0.842771 & -0.103112 & 1.000 & obsgroup \\
\hline
\end{tabular}

See file mod2.res for more details of residuals in graph-ready format.

See file mod2. seo for composite observation sensitivities.

Objective function ----->

Sum of squared weighted residuals (ie phi) $\quad=3.0829 \mathrm{E}-02$

Correlation Coefficient ----->

Correlation coefficient $\quad=0.9682$

Analysis of residuals ----->

All residuals:-

Number of residuals with non-zero weight

Mean value of non-zero weighted residuals

Maximum weighted residual [observation "c17"]

Minimum weighted residual [observation "c20"]

Standard variance of weighted residuals

Standard error of weighted residuals

$$
\begin{array}{lc}
= & 7 \\
= & -3.4076 \mathrm{E}-03 \\
= & 7.4902 \mathrm{E}-02 \\
= & -0.1031 \\
= & 6.1659 \mathrm{E}-03 \\
= & 7.8523 \mathrm{E}-02
\end{array}
$$

Note: the above variance was obtained by dividing the objective function by the number of system degrees of freedom (ie. number of observations with non-zero weight plus number of prior information articles with non-zero weight minus the number of adjustable parameters.) If the degrees of freedom is negative the divisor becomes 
the number of observations with non-zero weight plus the number of prior information items with non-zero weight.

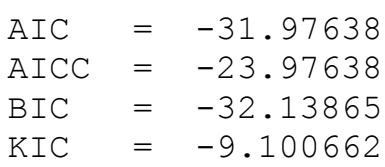

\begin{tabular}{lcr} 
& disp & \multicolumn{1}{c}{ vel } \\
disp & $1.7383 \mathrm{E}-08$ & $-7.9657 \mathrm{E}-08$ \\
vel & $-7.9657 \mathrm{E}-08$ & $1.1876 \mathrm{E}-06$
\end{tabular}

Parameter correlation coefficient matrix ----->

\begin{tabular}{lrr} 
& disp & \multicolumn{1}{c}{ vel } \\
disp & 1.000 & -0.5544 \\
vel & -0.5544 & 1.000
\end{tabular}

Normalized eigenvectors of parameter covariance matrix ----->

$\begin{array}{lll} & \text { Vector } 1 & \text { Vector } 2 \\ \text { disp } & 0.997 \overline{7} & -6.7600 \mathrm{E}-02 \\ \text { vel } & 6.7600 \mathrm{E}-02 & 0.9977\end{array}$

Eigenvalues ---->>

$1.1986 \mathrm{E}-08 \quad 1.1930 \mathrm{E}-06$ 